\title{
Insights into Nonalcoholic Fatty-Liver Disease Heterogeneity
}

\author{
Luca Valenti, MD ${ }^{4}$ Ariel E. Feldstein, MD $^{3}$ \\ ${ }^{1}$ Department of Gastroenterology, Escuela de Medicina, Pontificia \\ Universidad Católica de Chile, Santiago, Chile \\ ${ }^{2}$ Centro de Envejecimiento y Regeneración (CARE), Departamento de \\ Biología Celular y Molecular, Facultad de Ciencias Biologicas, \\ Pontificia Universidad Catolica de Chile, Santiago, Chile \\ 3 Department of Pediatric Gastroenterology, Rady Children's \\ Hospital, University of California San Diego, California \\ ${ }^{4}$ Department of Pathophysiology and Transplantation, Universita \\ degli Studi di Milano, Translational Medicine, Department of \\ Transfusion, Medicine and Hematology, Fondazione IRCCS Ca' \\ Granda, Pad Marangoni, Milan, Italy \\ Semin Liver Dis 2021;41:421-434.
}

Marco Arrese, $\mathrm{MD}^{1,2}$ Juan P. Arab, MD ${ }^{1,2}$ Francisco Barrera, MD ${ }^{1,2}$ Benedikt Kaufmann, MD ${ }^{3}$

\begin{abstract}
Address for correspondence Marco Arrese, MD, Department of Gastroenterology, Escuela de Medicina, Pontificia Universidad Católica de Chile, Diagonal Paraguay \#362, 8330077 Santiago, Chile (e-mail: marrese@uc.cl).
\end{abstract}

Ariel E. Feldstein, MD, Division of Pediatric Gastroenterology Hepatology and Nutrition, UCSD 3020 Children's Way, MC 5030, San Diego, CA 92103-8450

(e-mail: afeldstein@ucsd.edu).

\begin{abstract} Keywords

- nonalcoholic fattyliver disease

- steatosis

- cirrhosis

- fibrosis

- nonalcoholic

- steatohepatitis

- NAFLD

- NASH

- metabolic syndrome

- heterogeneity

The acronym nonalcoholic fatty-liver disease (NAFLD) groups a heterogeneous patient population. Although in many patients the primary driver is metabolic dysfunction, a complex and dynamic interaction of different factors (i.e., sex, presence of one or more genetic variants, coexistence of different comorbidities, diverse microbiota composition, and various degrees of alcohol consumption among others) takes place to determine disease subphenotypes with distinct natural history and prognosis and, eventually, different response to therapy. This review aims to address this topic through the analysis of existing data on the differential contribution of known factors to the pathogenesis and clinical expression of NAFLD, thus determining the different clinical subphenotypes observed in practice. To improve our understanding of NAFLD heterogeneity and the dominant drivers of disease in patient subgroups would predictably impact on the development of more precision-targeted therapies for NAFLD.
\end{abstract}

Nonalcoholic fatty-liver disease (NAFLD) is currently considered the commonest liver disease worldwide with an estimated global prevalence of $25 \%{ }^{1,2}$ The acronym refers to a range of hepatic histological alterations including isolated steatosis (also referred as NAFL), non-alcoholic streatohepatitis (NASH which is hallmarked by the presence of hepatocellular injury accompanied by inflammation and variable degrees of fibrosis), and cirrhosis. ${ }^{3}$ Importantly, NAFLD is a risk factor for hepatocellular carcinoma (HCC) development even in the absence of cirrhosis. ${ }^{4}$ NAFLD relevance is underscored by data pointing to NAFLD as the most fast-growing cause of cirrhosis and the need liver transplantation in the United States. ${ }^{5}$ Moreover, epidemiological figures are worrisome as, according to epidemiological modeling, NAFLD burden is expected to grow in the coming decades burdening health care systems and causing substantial economic costs and compromising individual health, determining significant morbidity and mortality. ${ }^{6}$

To date, dietary caloric restriction and exercise are the cornerstone of NAFLD therapy, and although these measures have been proven to be efficacious, therapeutic goals are difficult to achieve or sustain. ${ }^{7-9}$ Unfortunately, no Food and Drug Administration (FDA)-approved therapies for NAFLD published online

July 7, 2021
DOI https://doi.org/ 10.1055/s-0041-1730927. ISSN 0272-8087. (c) 2021. The Author(s).

This is an open access article published by Thieme under the terms of the Creative Commons Attribution-NonDerivative-NonCommercial-License, permitting copying and reproduction so long as the original work is given appropriate credit. Contents may not be used for commercial purposes, or adapted, remixed, transformed or built upon. (https://creativecommons.org/ licenses/by-nc-nd/4.0/)

Thieme Medical Publishers, Inc., 333 Seventh Avenue, 18th Floor, New York, NY 10001, USA 
are currently available. Clinical trials conducted so far with a myriad of investigational agents have shown relatively low rates of response compared with placebo. ${ }^{10}$ The latter may be related to several factors ${ }^{11}$ with disease heterogeneity being a relevant aspect. In fact, inclusion criteria for most of clinical studies is usually based mainly on histological diagnosis of NAFLD which result in mixed population of patients. $^{12}$ Thus, more granular data on the effectiveness of new therapies in NAFLD subgroups is needed to create the proper grounds for personalized or precision medicine approaches in the near future. ${ }^{13-15}$

As mentioned above, the NAFLD acronym groups a heterogeneous patient population. NAFLD clinical expression results from a complex and multilayered dynamic interaction of different factors including sex, ${ }^{16}$ presence of several genetic variants, ${ }^{17}$ coexistence of different comorbidities, ${ }^{18}$ diverse microbiota composition, ${ }^{19-21}$ and various degrees of alcohol consumption $^{22}$ among others. This complex interaction results in several disease subphenotypes with distinct natural history and prognosis and, eventually, different response to therapy ( $\mathbf{- F i g . 1}$ ). Of note, NAFLD heterogeneity was one of the reasons argued by an expert panel that suggested to replace NAFLD by a new acronym derived from the initial letters of metabolic (dysfunction)-associated fatty-liver disease (i.e., MAFLD). NAFLD renaming was proposed aiming to better capture the main driver of the disease in the majority of patients. ${ }^{23,24}$ However, this suggestion had not been universally accepted and a debate on the matter is ongoing. ${ }^{25,26}$ In the present review, we aim to address the issue of NAFLD heterogeneity through the analysis of existing data on the differential contribution of known factors to the clinical expression of NAFLD. The impact of better deciphering NAFLD heterogeneity and the dominant drivers of disease in patient subgroups on the development of more targeted therapies for NAFLD is also highlighted.

\section{Role of Genetic Determinants}

NAFLD has a strong heritability and during the last year's genome-wide association studies (GWAS) have allowed the discovery of the main common genetic determinants of this condition and of the susceptibility to hepatic fat

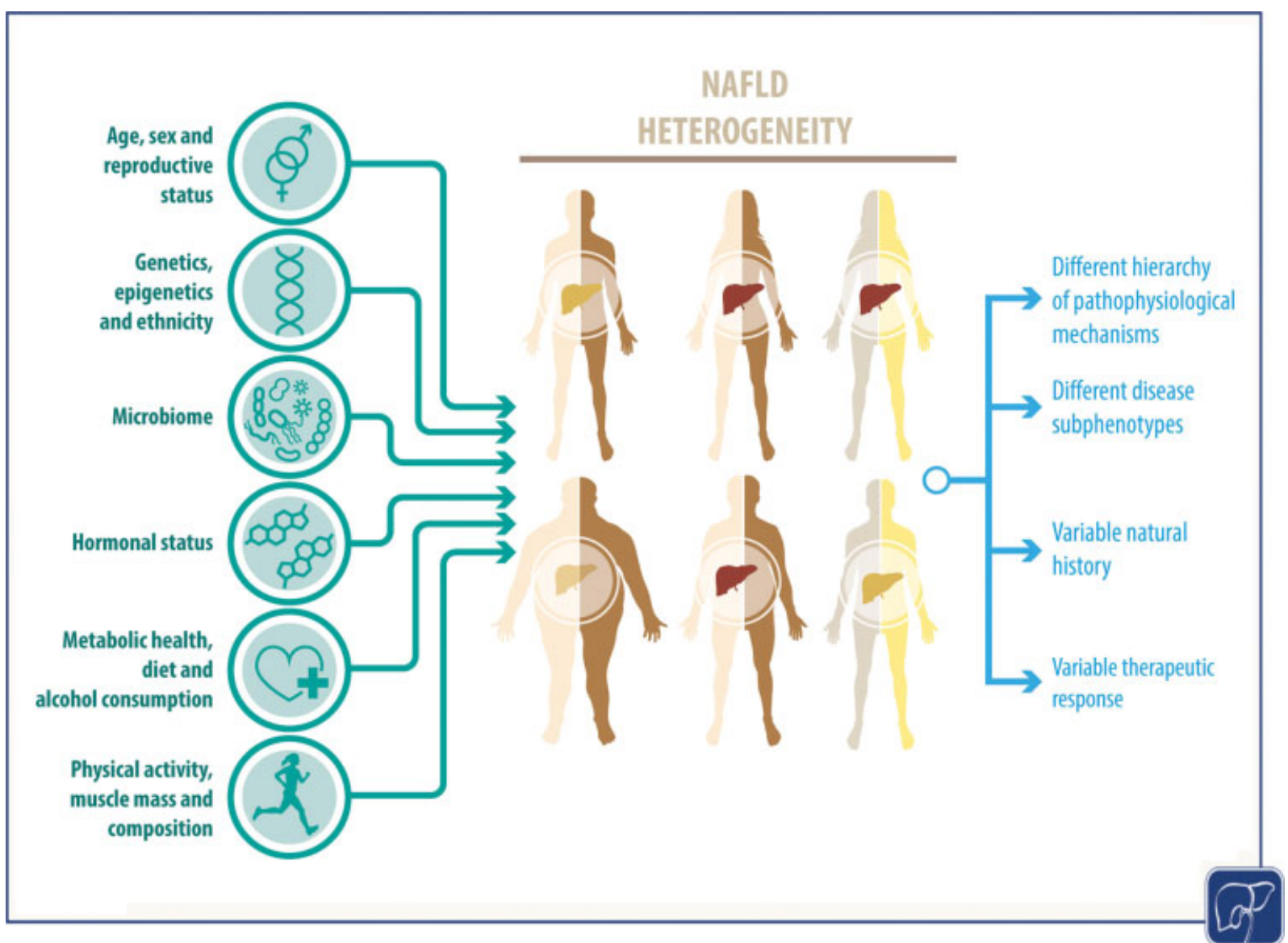

Fig. 1 The acronym nonalcoholic fatty-liver disease (NAFLD) groups a heterogeneous patient population. The different phenotypes observed in clinical practice stem from a complex and dynamic interaction of different factors (age, sex, reproductive status, presence of one or more genetic variants and epigenetic factors, a diverse microbiota composition, coexistence of different comorbidities, the degree of alcohol consumption, and muscle mass and physical activity among other). These variables critically influence NAFLD development and progression. Disease heterogeneity imply that pathophysiological mechanisms underlying NAFLD may have different hierarchy or trajectory in different patients defining different subphenotypes which may be relevant to molecular pathway-based therapies. NAFLD heterogeneity may also influence natural history and prognosis and, eventually, response to therapy. 
accumulation. ${ }^{17}$ Their common pathogenic mechanism turned out to be the alteration of hepatocellular lipid handling, favoring on one hand retention in lipid droplets over secretion within very-low density lipoproteins and on the other hand synthesis over catabolism. ${ }^{27}$ Increased quantity and altered composition of lipids determines lipotoxicity and potentially progressive liver disease. ${ }^{27}$

Identification of patatin-like phospholipase domain-containing 3 (PNPLA3) p.I148M and transmembrane 6 superfamily member 2 (TM6SF2) p.E167K, as the main risk variants, allowed soon to pinpoint a subset of patients carrying multiple variants who are at higher risk of progression to the hepatic rather than to the cardiometabolic complications of NAFLD. ${ }^{14,17,28,29}$ Due to the increased power of studies conducted in large population-based cohorts, the number of NAFLD risk variants is now increasing by the day. ${ }^{30-33}$ We are therefore presently moving to a model where, across the continuum of the distribution of the inherited predisposition to NAFLD in the population, the development of polygenic risk scores (PRS) can stratify the risk of this condition and of liver-related complications. $^{30,31,34-36}$ For example, "high" PRS can predict the evolution to cirrhosis and HCC independently of classical risk factors in individuals with dysmetabolism and/or NAFLD, potentially being able to aid in the clinical management and in the design of referral pathways. ${ }^{35}$

On the other hand, it has become clear that the main common risk variant and PRS cannot discriminate accurately between "metabolic" and "genetic" NAFLD. ${ }^{28}$ Vice versa, very robust evidence has accumulated that increased adiposity, and dysmetabolism is the main trigger for the phenotypic expression of this genetic predisposition; the higher the body mass index, the higher the risk of liver disease in carriers of NAFLD risk variants. ${ }^{30,35,37,38}$ In addition, genetic predisposition to NAFLD contributes to insulin resistance via inducing liver injury. ${ }^{34}$ Finally, the main genetic risk variants in PNPLA3 and TM6SF2 are similarly enriched in "overweight/obese" versus "lean" NAFLD. ${ }^{39,40}$ Indeed, central adipose tissue distribution, genetic causes of insulin resistance, ${ }^{37}$ and other factors predisposing to hepatic fat accumulation can vicariate the role of excess adiposity among the small fraction of lean individuals who develop NAFLD.

Excess intake of alcohol is another key determinant of the phenotypic expression of NAFLD risk variants ${ }^{17,30}$ which are also the main genetic risk factors of alcoholic liver disease. ${ }^{41}$ Fructose intake, chronic hepatitis $\mathrm{C}$, and iron accumulation are additional triggers of liver disease. ${ }^{42-44}$ This new evidence suggests that several conditions that were previously considered as separated diseases share major pathogenic mechanism of liver damage centered on fat accumulation and lipotoxicity which are initiated by specific but often concomitant insults. The most frequent is the combination of obesity, excessive alcohol, and genetic predisposition. Environmental and toxic insults may further push this deleterious mix toward liver disease. As such, genetics has clearly highlighted that clinical heterogeneity should perhaps be the best considered in the overall context of fatty-liver diseases than in NAFLD per se.
However, there is initial evidence from the first next generation sequencing studies that rare genetic variants with a large impact on hepatic fat can predispose to NAFLD irrespective of dysmetabolism. ${ }^{14,45}$ For example, carriage of apolipoprotein $\mathrm{B}(\mathrm{APOB})$ variants predispose to severe NAFLD, cirrhosis, and HCC in nonobese individuals. ${ }^{45,46}$ This subset of patients is at low risk of cardiovascular disease due to reduced circulating lipoproteins and may also possibly benefit from vitamin E supplementation due to malabsorption of lipophilic vitamins. ${ }^{45-47}$

There is additional evidence that the genetic setup of NAFLD can influence the profile of extrahepatic organ damage and the response to therapy. Thanks to the recent discoveries in NAFLD genetics, ${ }^{14,17,28,29}$ the frontier of disease phenotyping can move away from the "metabolic vs. genetic" simplistic dichotomy, toward a subclassification integrated with the clinical risk factors and based on combined environmental-genetic pathways underlying disease predisposition. The challenge ahead will be to develop reliable clusters of genetic variants that predict disease subtypes. Based on the current knowledge at least three groups can be identified. The first is defined by the presence of high genetic risk of obesity and insulin resistance ${ }^{37,48}$ in the absence of specific risk determinants of liver injury ("insulin-resistant fatty-liver disease"). This roughly corresponds to the previous classification of "metabolic NAFLD," at high risk of cardiometabolic complications, but lower risk of liver-related events. Among the specific determinants of liver involvement in individuals with dysmetabolism, a group of genetic variants including those in glucokinase regulator $(G C K R)$ that facilitate fat accumulation by diverting carbon substrates from glucose metabolism to lipid synthesis (de novo lipogenesis [DNL]) can define "enhanced lipogenesis fatty-liver disease," at high risk of dyslipidemia. ${ }^{17,49}$ Insulin-resistant patients carrying these variants may possibly benefit from approaches reducing lipogenesis, such as statins and omega-3 fatty acids. ${ }^{14,50}$ A pathophysiological distinct subset ("impaired lipid remodeling fatty liver disease") is represented by those with a high burden of genetic variants, including those in PNPLA3, TM6SF2, membrane bound O-acyl transferase (MBOAT7), and $A P O B$, impairing lipid remodeling and secretion and favoring lipotoxicity. These patients are at a particularly high risk of liver-related complications, including hepatocellular carcinoma before cirrhosis development. ${ }^{14}$ They develop type-2 diabetes, ${ }^{34,48}$ but are relatively protected from cardiovascular disease. ${ }^{14,35}$ From a therapeutic point of view, they respond well to weight loss, but when they carry the PNPLA3 variant do not benefit from statins and are at increased risk of liver injury due to enhanced hepatic lipid accumulation during insulin therapy (reviewed by Cespiati et $\mathrm{al}^{14}$ ).

Overall, the newest research developments have suggested new possibilities to integrate, rather than oppose the genetic markers with clinical information to improve fatty-liver disease classification and management. The efficacy and cost effectiveness of this approach remains to be proven before wide implementation can be advised. 


\section{Differential Contribution of Pathogenetic Pathways}

NAFLD pathogenesis is associated with a wide spectrum of aberrant cellular and molecular mechanisms. ${ }^{51-53}$ As described above, genetic ${ }^{54}$ and metabolic ${ }^{55}$ alterations are key drivers of the development of NAFLD resulting in an environment that favors hepatic fat accumulation and toxic lipid metabolites via an increase in circulating free fatty acids released from a dysfunctional inflamed adipose tissue ${ }^{56,57}$ and an increased DNL in hepatocytes. ${ }^{58,59}$ These changes may lead to a state of "lipotoxicity" that triggers hepatocellular stress and consequently may result in liver injury and cell death, activating a sterile liver inflammatory response ${ }^{51,53,60}$ and ultimately liver fibrogenesis contributing to disease progression. The mechanisms thought to be at play in NAFLD pathogenesis may act in a sequential or parallel fashion and with different hierarchies within the spectrum of disease and over time. ${ }^{57,60}$ Thus, it is likely that the observed heterogeneity of NAFLD in the clinic, where some patients develop isolated steatosis, whereas others develop NASH and advanced chronic liver disease stems from a differential contribution of pathogenetic pathways, ${ }^{60,61}$ as well as from the activation or deactivation of redundant mechanisms. Also, it is likely that, besides genes, many factors including diet, exercise, sex, age, and coexistence of obesity and type 2 diabetes mellitus (T2DM) among others, critically influence disease pathogenesis in a given patient. ${ }^{15}$ However, to dissect the precise contribution of each pathway to NAFLD development and progression is difficult due to limitations of available animal models ${ }^{62,63}$ or lack of robust human data. Remarks on potentially relevant pathogenic differences among NAFLD patients are provided below.

One important mechanism underlying NAFLD development is an upregulated DNL which has been reported to be three-fold increased compared with control patients. ${ }^{58}$ Interestingly, DNL rates are influenced by age and sex, and this pathway may be pathogenically more relevant in older and male patients than others. ${ }^{64,65}$

A previously overlooked player in NAFLD pathogenesis is the skeletal muscle. Several studies have underscored that sarcopenia (i.e., decreased muscle mass) or adverse muscle composition (i.e., low muscle volume and muscle fat infiltration or myosteatosis) may influence both NAFLD development and progression. ${ }^{66-68}$ Although important methodological differences exist across published studies, not all patients with NAFLD exhibit muscle alterations and it is likely that this pathogenic factor be more relevant in particular populations, such as nonobese or lean NAFLD, as well as older patients in which sarcopenia is more frequent. ${ }^{69}$

Activation of the innate immune system has been implicated in NAFLD progression. ${ }^{70,71}$ After liver damage, a physiological immune response is key for resolution of the injurious process, liver regeneration and restauration of a healthy liver state, while an exuberant and persistent innate immune response may lead to chronic liver inflammation. In the context of NAFLD, an imbalance of the cross-talk of the gut microbiome and the liver, known as the gut-liver axis, as well as occurrence of hepatocyte cell injury and death are two key upstream triggers of a persistent pathogenic innate immune response. ${ }^{57,71}$ Intestinal dysbiosis, defined as the imbalance between resident microbial communities that disturb the symbiotic relationship between gut microbes and the host, and an increased intestinal permeability may lead to a pathological translocation of microbial products to the liver via the portal vein. ${ }^{19,20}$ In this setting, pathogenassociated molecular patterns (PAMPs) are recognized by selective pattern recognition receptors, mainly toll-like receptors, in the liver and cause a chronic innate immune response. In addition, microbiota-derived metabolites such as modified bile acids, choline, and ethanol alter hepatic metabolism and trigger an inflammatory response. ${ }^{72}$ The above-mentioned mechanisms may be at play in only a fraction of individuals with NAFLD. In fact, clinical studies have uncovered that only a subset of NAFLD patients present with dysbiosis and/or a dysfunction in the gut barrier. ${ }^{19,72}$ These findings point that in addition to the genetic and metabolic modifiers, assessment of microbiome profile, and or intestinal permeability may allow for a more precise classification and risk stratification of NAFLD patients to define an adequate therapeutic intervention. ${ }^{72-74}$

In certain settings, the immune system may be activated by other stimuli and contribute to NAFLD development and progression. This may be the case for the association of NAFLD with some immune-mediated inflammatory disorders, such as psoriasis ${ }^{75,76}$ and suppurative hidradenitis, ${ }^{77}$ which are associated to a low-grade systemic inflammatory state. Of note, Psoriasis patients exhibit significantly increased serum levels of proinflammatory cytokines such as tumor necrosis factor-a (TNF-a) interleukin (IL)-6 and reduced levels of the anti-inflammatory adipokine, adiponectin than matched controls. ${ }^{78}$ Also, psoriasis patients with NAFLD have higher serum levels of C-reactive protein (CRP) and have an increased prevalence of advanced liver fibrosis. ${ }^{79}$ In the same line, patients with suppurative hidradenitis exhibit a high prevalence of NAFLD independently of classic metabolic risk factors. ${ }^{80}$ However, it remains difficult to dissect whether the underlying systemic inflammation observed in psoriasis and suppurative hidradenitis is a major contributor to NAFLD development and progression given the frequent coexistence of obesity in these patient populations. ${ }^{76,81}$ Interestingly, an imbalance in T-cell subtypes, like Th17/Treg, leading to an IL-17-mediated inflammation may be a common pathogenic link in this regard. ${ }^{76,82}$

Hepatocyte cell death as a result of lipotoxicity is another key driver of the progression of NAFLD. Cellular release of damage-associated molecular patterns (DAMPs) by damaged $^{71}$ or stressed hepatocytes are recognized by the innate immune system and may trigger chronic liver inflammation. ${ }^{60,83}$ Recently pyroptosis has been identified as a novel form of programmed cell death in NAFLD and NASH. Pyroptosis is characterized by the activation and assembly of multiprotein complexes, called inflammasomes. The most studied one is the nucleotide-binding domain, leucine-richrepeat (NLR) family, pyrin domain containing 3 (NLRP3) inflammasome. Once activated, the NLRP3 inflammasome 
cleaves procaspase- 1 to its mature form caspase- 1 that subsequently cleaves IL-1 $\beta$, IL-18, and gasdermin D. IL-1b and IL-18 are highly proinflammatory cytokines that are released into the extracellular space partly through gasdermin D formed transmembrane pores. ${ }^{60,84}$ NLRP3 was shown to promote NASH and liver fibrosis in various mouse models. Genetic constitutive overactivation of the NLRP3 inflammasome causes pyroptosis, inflammation, and fibrosis in the liver, while global NLRP3 knock out mice show less inflammation and liver fibrosis in models of diet induced NASH. ${ }^{85,86}$ Human data on the contribution of NLRP3 to NAFLD development and progression is scarce; thus, future translational studies to examine the human relevance of these pathways to NAFLD heterogeneity are warranted.

As described above, PAMPs and DAMPs activate and sustain a pattern recognition receptor-mediated innate immune response characteristic for NAFLD. Once activated, a complex intercellular cross-talk between the different innate immune cells, mainly macrophages, neutrophils, natural killer cells, and T-cells, as well as hepatocytes and hepatic stellate cells (HSCs), defines the progression to steatohepatitis and fibrosis. Macrophages are known to play a crucial role in orchestrating immune response and the recruitment of further immune cells. ${ }^{87}$ Kupffer's cells, the resident liver macrophages, are dominant over monocytes and monocytederived macrophages (MoMFs) in a healthy liver. However, after liver injury, both monocytes and subsequently MoMFs are rapidly recruited to the liver. ${ }^{88}$ The functional plasticity of macrophages was historically subclassified in the two polarizations, proinflammatory-type M1 and anti-inflammatory-type M2. This classification was shown to lack accuracy of the macrophage activation program. Instead, during liver injury, macrophages display wide gene expression profiles that led to the identification of different subpopulations of macrophages. The new technology of singlecell RNA sequencing (scRNA-seq) revealed an accumulation of MoMF in murine livers in a diet-induced NASH model. ${ }^{89}$ The population of MoMF was further subclassified in the three clusters MoMF I to III with each cluster characterized by particular marker genes. Interestingly, similar subpopulations of bone marrow monocytes were also detected. These findings indicate a NAFLD gene signature in myeloid leukocytes in both liver and bone marrow. ${ }^{88}$ Another study using scRNA-seq identified further subpopulations of macrophages in NASH characterized by the expression of Trem2, termed NASH-associated macrophages. In human samples, Trem2 expression correlated with the severity of NAFLD activity score and the extent of fibrosis. Therefore, markers of NASH-associated macrophages may function as potential novel key classifiers of NAFLD/NASH phenotypes. ${ }^{90}$ The specific role of Kupffer's cells during the progression of NAFLD to NASH has continued to evolve. Recent evidence has uncovered a reduction of mature Kupffer's cell population during this process due to increased Kupffer's cell death. Monocyte-derived macrophages are then able to enter the stage of differentiation into Kupffer's cells. While Kupffer's cell acts mainly by facilitating hepatocyte triglyceride accumulation, MoMFs are more proinflammatory. ${ }^{91,92}$ A better understanding and profiling of the immune cell niche during NAFLD and NASH may provide a precision medicine approach to the current pathological scoring of inflammation and allow for risk stratification and identify patients more likely to have a progressive disease versus those who will have a nonprogressive liver phenotype.

Liver fibrosis is the critical pathological feature that predicts liver-related outcome in NASH. ${ }^{93,94}$ Various cytokines activate HSCs and their transformation toward collagen secreting myofibroblasts (MFB) which are key for triggering and progression of liver fibrosis as in other hepatic diseases. ${ }^{95}$ However, considering the disturbed metabolic milieu present in NAFLD, it is likely, although not certain that a myriad of disease-specific mechanisms may be at play (recently reviewed by Schwabe et al). ${ }^{96}$ Interestingly, a recent study investigating the population of activated HSCs in liver fibrosis revealed various clusters of MFBs, suggesting the existence of heterogeneity within the fibrogenic cell population. While all clusters showed a high expression of collagen, the ability to secret chemokines to modulate inflammation was limited to certain clusters. ${ }^{97}$ Another study uncovered specific liver zonation of HSCs, classifying them into portal vein-associated HSCs (PaHSCs) and central vein-associated HSCs (CaHSCs). Notably, CaHSCs were identified as the dominant pathogenic collagen-producing cells. The lysophosphatidic acid receptor 1 (LPAR1) is upregulated in NASH patients and selectively expressed in CaHSCs but not PaHSCs and NPCs. Pharmacological inhibition of LPAR1 in a murine NASH model has shown to ameliorate liver fibrosis. ${ }^{98}$ These findings underline the complexity and diversity of HSCs in the context of the pathogenesis of liver fibrosis in general and in NAFLD in particular. ${ }^{96}$ Further studies are warranted to investigate whether certain subpopulations of HSCs favor remodeling after liver injury or triggering liver fibrosis. These studies may allow to identify patients with fibrotic NASH that are at particular risk of worsening of fibrosis versus those that are more likely to show a robust wound healing response with potential for spontaneous improvement of fibrosis. In summary, these novel studies dissecting the distinct immune cell and HSC niche may allow to provide two additional groups to the ones described in the previous section including an "Inflammatory fatty liver disease" and a "profibrotic fatty-liver disease." This classification may be central to identify patients with NAFLD/NASH that are at particular risk for disease progression or "rapid progressors," a subphenotype that represents the central target for therapeutic intervention.

\section{Heterogeneity of Clinical Disease}

In the clinical arena, NAFLD patients present with diverse features. Sources of heterogeneity that can be considered in the clinic at present time are multiple and include age, sex, cardiometabolic comorbidities, ethnicity, endocrine status, as well as alcohol and tobacco consumption. $^{23}$

Considerations on the influence of age and sex in NAFLD clinical presentation and prognosis has been reviewed elsewhere, ${ }^{99-101}$ underscoring that while pediatric patients may 
have a different natural history and unique susceptibilities, as well as more severe long-term consequences, ${ }^{101}$ older patients exhibit an increased prevalence and severity of NAFLD. ${ }^{102}$ With regard to sex, NAFLD is considered a sexually dimorphic disease with clear differences between male and females with the latter being protected of severe fibrotic disease during premenopausal stage. ${ }^{103}$

Being a multisystem disease, NAFLD is commonly associated with metabolic features, with a prevalence of $80 \%$ of obesity, $72 \%$ dyslipidemia, and $44 \%$ of T2DM. ${ }^{1,104}$ Although the combination of these features acts in an additive manner to promote NAFLD progression, T2DM is the feature most strongly and consistently associated with a more severe disease and progression. ${ }^{105-107}$ Considering the ominous effect of T2DM on liver disease progression and extrahepatic complications (i.e., cardiovascular disease, cancer risk, and chronic kidney disease), most guidelines recommend patients that NAFLD need to be screened and treated for metabolic comorbidities. ${ }^{3,108,109}$ In addition, while obesity is also considered a risk factor for disease progression, ${ }^{107,110}$ lean patients with NAFLD have been increasingly recognized as being at risk of progression to advanced liver disease, as well as of developing metabolic comorbidities, and cardiovascular disease with impact in overall and liver-related mortality. ${ }^{111,112}$ Lean patients account for 20 to $30 \%$ of NAFLD patients. ${ }^{113}$ The same as obese NAFLD, its presence is associated with presence of metabolic comorbidities, increased visceral adiposity and unfavorable genetic polymorphisms. ${ }^{114}$ Of note, lean NAFLD is not a benign condition, since it is independently associated with increased overall and cardiovascular mortality. ${ }^{115}$

Ethnicity is an important aspect in NAFLD heterogeneity. Several studies describe Hispanic Latino population to be at the highest risk for development of NAFLD, whereas nonHispanic Black and non-Hispanic Asian population are at lowest risk. ${ }^{116}$ In the United States, most of this risk is explained specifically by Mexican American population (50\% NAFLD prevalence, compared with only $30 \%$ of prevalence in other Hispanic populations). The cause for these ethnicity differences is probably explained by genetic and sociocultural factors including diet, exercise, alcohol consumption, education, family income, and quality of life. Interestingly, novel data suggest that advanced fibrosis assessed by noninvasive serum test is more prevalent in non-Hispanic black (28.5\%) and non-Hispanic white populations (25.8\%) compared with Mexican American (10.8\%) and Asian non-Hispanic population (2.65\%). ${ }^{117}$ Indeed, further studies are needed to validate and explore these findings.

Sex differences is another important factor to be considered in NAFLD heterogeneity. It has been repeatedly shown that female patients at reproductive age have a significantly lower NAFLD prevalence and severity ${ }^{118}$ which may be explained by a myriad of sex differences in the pathobiology of NAFLD (e.g., different storing of surplus calories, differential activation of hepatic DNL, and sex-specific in mitochondrial function and immune responses among others). ${ }^{99}$ After menopause, sex differences are reduced in NAFLD and, in fact, women have a higher risk of advanced fibrosis than men, especially after age 50 years, ${ }^{119}$ suggesting that estrogen could be a protector factor for NAFLD. ${ }^{99,118}$ Again, sociocultural characteristics are likely to be involved in sex differences in NAFLD risk.

Obstructive sleep apnea (OSA) is a common complication of obesity, inducing repetitive cycles of hypoxia and reoxygenation. Presence of OSA is associated with increased liver steatosis, inflammation, and fibrosis. ${ }^{120,121}$ Unfortunately, trials evaluating OSA therapy with continuous positive airway pressure (CPAP) have shown nonconclusive results in reducing liver fibrosis of NAFLD patients. $^{120,122}$

Alcohol consumption has classically been considered an exclusion criterion for NAFLD. However, it has been increasingly recognized that a group of patients may present a dual etiology (i.e., alcoholic and nonalcoholic) of their fatty-liver disease. $^{22}$ Recent population based longitudinal studies demonstrate that even mild consumption of alcohol can have a synergistic effect when combined with obesity, insulin resistance, and metabolic dysfunction to increase risk of cirrhosis and hepatocellular carcinoma. ${ }^{22}$ In this context, the renaming of NAFLD into MAFLD proposes a set of positive criteria for diagnosis and does not consider the exclusion of alcohol consumption as a prerequisite criterion for diagnosis. $^{23,24}$

Among other relevant factors that may modulate disease development and severity are presence of skin inflammatory disorders, such as psoriasis and suppurative hidradenitis, hypothyroidism, polycystic ovarian syndrome, and periodontitis, all of which have been associated with increased prevalence of NAFLD. ${ }^{123}$

Besides systemic comorbidities, patients with NAFLD represent a wide spectrum of liver disease severity. In the previous decade, emphasis was made in a dichotomic differentiation between those patients with isolated steatosis and those with NASH. More recently, emphasis is made on patients with NASH and liver fibrosis as the latter histological feature has been shown to be a major prognostic determinant in NAFLD patients. ${ }^{94}$ Natural history studies, as well as studies examining patients on the placebo arms of drug clinical trials, using paired biopsies, showed that evolution of NAFLD/NASH overtime is highly dynamic and that, although NASH has a higher risk of fibrosis progression and development of cirrhosis than non-NASH patients, some individuals will have spontaneous improvement while some patients with isolated steatosis could also progress to NASH and cirrhosis. ${ }^{124-131}$ Current data support the idea that approximately 20 to $25 \%$ of patients with isolated steatosis will progress to NASH, and from those, approximately 20 to $40 \%$ will progress to advanced fibrosis and cirrhosis. Additionally, 5 to $7 \%$ of NASH-cirrhotic patients will develop HCC. ${ }^{56}$ Thus, identifying the group of patients who most likely will progress to advanced fibrosis is a primary goal of NAFLD/NASH-related research. In this regard, those at higher risk are patients with metabolic abnormalities, such as T2DM, metabolic syndrome, and obesity. ${ }^{2,132}$ 
Most natural history studies of NAFLD have assessed liver-related morbidity and mortality in relation to a single time-point evaluation of histology to identify risk factors. ${ }^{124,131,133-135}$ In spite of their intrinsic selection bias, paired-biopsy studies are informative regarding identification of factors associated with disease progression. ${ }^{125,126,128-130}$ In these studies, the main risk factors for presence of NAFLD are metabolic abnormalities such as T2DM, metabolic syndrome, and obesity. ${ }^{136-138}$ Regarding predictors of fibrosis progression, studies have shown that the best predictors are histological parameters including hepatocellular ballooning degeneration and inflammation associated with age in patients with NASH. ${ }^{130}$ As mentioned above, the presence of fibrosis on initial biopsy or progression of fibrosis on serial biopsies appears to be the most prominent histologic predictor of overall mortality and liver-related outcomes. ${ }^{139-141}$ During the last decade, efforts have been made to identify noninvasive surrogates of liver biopsy that can be followed over time. Serum aminotransferase levels are good predictors of improvement of NASH in clinical trials. ${ }^{10}$ Likewise, markers of cell death including soluble cytokeratin-18 (CK-18) forms (total and fragmented) have been shown to be good diagnostic markers of NASH and predict response to therapeutic interventions but currently remain a research tool and are not clinically available. ${ }^{142,143}$ Other circulating markers such as proinflammatory markers, including circulating levels of cytokines (onlinTNF-a, IL-6, IL-8, and CRP), or other molecules, such as adiponectin, have shown inconsistent results in part due to the challenges with the sensitivity of the assays used to detect changes in blood. ${ }^{143-146}$ To identify patients who already are progressing and patients at early stages of fibrosis, noninvasive scores have been developed. Among them, the NAFLD Fibrosis Score, Fibrosis-4 (FIB-4), and Hepamet are nonexpensive, noncommercial, and easily available. ${ }^{147-152}$ The enhanced liver fibrosis (ELF) test is a commercial panel that combines various constituents of collagen matrix deposition and turnover. $^{153}$ Additionally, imaging assessment of steatosis and fibrosis has been developed as a noninvasive method and has focused mainly on transient elastography (TE) with controlled attenuation parameter (CAP) and magnetic resonance imaging (MRI)-based technologies. ${ }^{153-158}$ Details on modern concepts about noninvasive assessment of hepatic fibrosis can be found in recent in-depth reviews on the topic. ${ }^{159,160}$

\section{Implications for Clinical Practice and Research}

The differential contribution of genetic/epigenetic, environmental, and metabolic factors deters a significant interpatient variation regarding the major driver of disease (-Fig. 2). Proper consideration of NAFLD heterogeneity is relevant for both clinical practice and research. In the clinical arena, a more precise patient phenotyping could allow to a more granular grouping of patients to better stratify them into those at higher risk of adverse outcomes. Also, a better phenotyping would allow the implementation of tailored treatment approaches in line with concepts of precision medicine or individualized care. ${ }^{13,15,161}$ Since the degree of liver fibrosis has been shown to be closely related to liverrelated mortality, ${ }^{93,94}$ assessment of this variable is now considered crucial in patient stratification and should be performed with the available noninvasive tools. ${ }^{159}$ However, most of these tools are not able to distinguish those patients that show a strong wound healing response and thus more likely to have a resolutive phenotype with spontaneous regression of fibrosis versus those that will have a strong fibrogenic response and will more likely show progression of fibrosis. The challenge for the future will be to develop deep phenotyping approaches that also takes into consideration these processes in a given patient, and incorporates associated comorbidities, race, genetic and epigenetic influences, and environmental factors. ${ }^{10,162}$ Thus, clinical variables known to be associated with rapid disease progression, such as T2DM, arterial hypertension, severe obesity, and worsening metabolic health (i.e., presence of disglycemia and having one or more components of metabolic syndrome), should be factor in association with molecular and cellular profiling of liver phenotypes and if present, may confer the label of "high-risk" NAFLD/NASH ${ }^{163,164}$ which may imply higher risk of all-cause mortality, as well as of fibrosis, cirrhosis, and HCC. ${ }^{165}$ In addition, consideration on differences linked to age, sex, and hormonal status may be proven relevant given that these factors may influence NAFLD natural history, as well as the performance of several diagnostic tools. ${ }^{15}$ With regard to genetic factors, routine genotyping of genetic variants known to influence NAFLD has not yet been proven to be cost effective but likely will contribute to individualized management when used integrated with all pertinent clinical information ${ }^{14,166}$ (see section Role of Genetic Determinants). Finally, detailed phenotyping of behavioral (i.e., physical activity and diet), alcohol use, and socioeconomic factors, as well as of muscle mass and composition (i.e., using MRI techniques), could aid in better defining NAFLD patient cohorts.

From a therapeutic standpoint, NAFLD heterogeneity should be considered when structuring a therapeutic plan for disease management. As previously outlined in several clinical guidelines or position papers released and endorsed by several scientific societies or expert panels, ${ }^{3,108,167}$ lifestyle modifications remain the cornerstone of NAFLD management and are indicated in all patients. ${ }^{7}$ Of note, sex differences in the response to lifestyle changes have been observed in several studies. Interestingly in the most-cited study assessing lifestyle interventions in NAFLD, ${ }^{168}$ men showed a greater histological improvement than women after weight loss. In this study, male sex was one of the factors predicting beneficial histological response following a relatively modest weight loss (between 7 and 10\%), while in women, a more substantial weight loss (>10\%) was required to achieve a significant histological improvement. ${ }^{168,169}$ Thus, based in these observations, women may require additional support to achieve the goals to be achieved after introduction of lifestyle changes. In contrast, patients carrying the p.I148M variant of the PNAPL3 gene exhibit a better response to lifestyle modification and bariatric surgery than patients not carrying the variant. ${ }^{170-172}$ This information may be of 


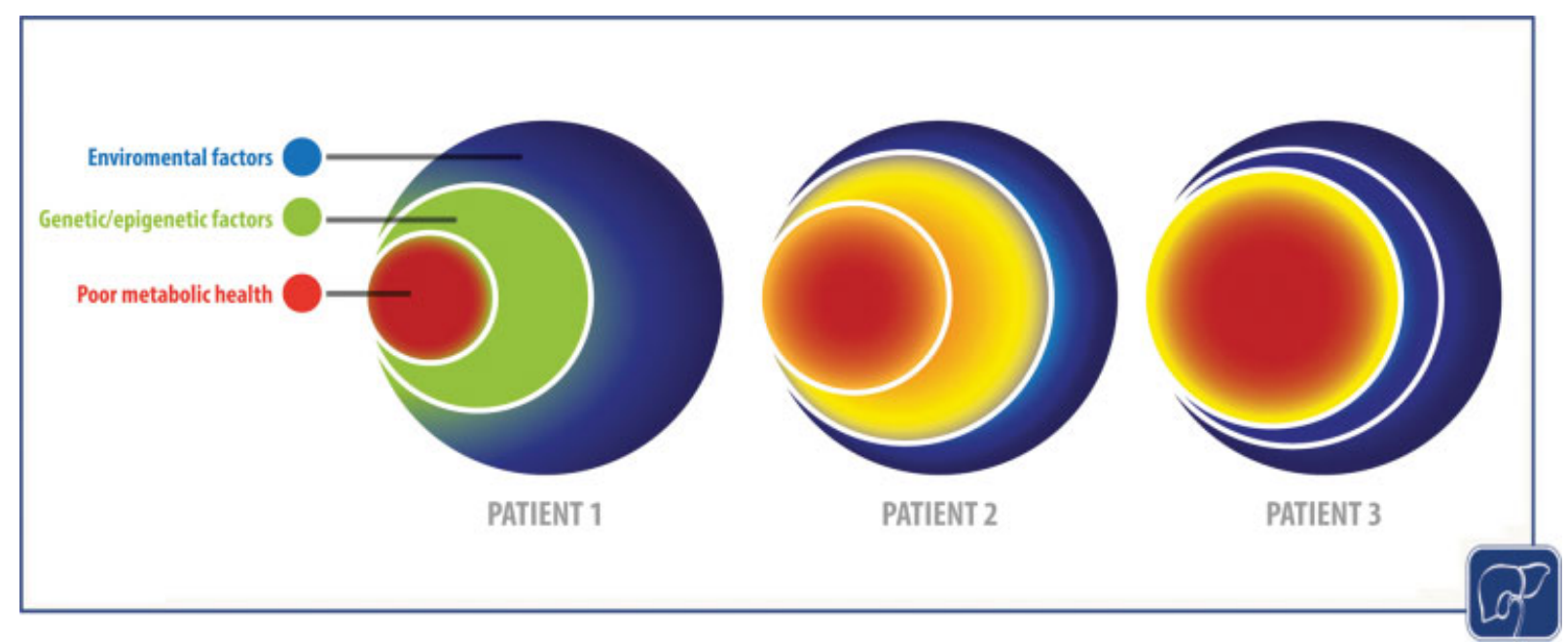

Fig. 2 The differential contribution of genetic/epigenetic, environmental and metabolic factors deters a significant interpatient variation regarding the major driver of disease. Circles depict three hypothetical patients that exhibit a different predominance of the three abovementioned factors. While patient 1 has environmental factors (e.g., poor diet and/or physical activity) as main driver of disease, patients 2 and 3 has a mixed predominance of genetic factors and metabolic derangement as determinants of their phenotypes. Better distinction of main drivers in each patient may help to implementation of individualized or precision medicine approaches in nonalcoholic fatty-liver disease (NAFLD) management (adapted from Eslam et al). ${ }^{23}$

aid when considering obesity surgery in NAFLD patients. The same applies to the possibility of applying tailored cardiovascular risk management in patient subgroups as patients with the p.I148M variant of PNAPL3, as well as those with the TM6SF2 E167K gene variant, exhibit a lower risk of cardiovascular disease compared with noncarriers. ${ }^{17}$ Finally, tailored HCC screening may be envisioned for some patients using genetic information as use of PRS, as shown by Bianco et al in a recent paper in which PNPLA3-TM6SF2GCKR-MBOAT7 were combined, may improve HCC risk stratification in NAFLD. ${ }^{173}$

With regard to interpatient variability to therapeutic agents, available information is scarce. It is likely that better and more detailed assessment of certain specific pathways could be of help in selecting therapeutic agents. This could be made with the use of metabolomics ${ }^{174,175}$ or other system biology tools. Thus, if DNL is shown to be overactive, ${ }^{65}$ specific use of agents targeting this pathway, such as acetyl-CoA carboxylase (ACC) inhibitors, could be indicated. ${ }^{174,175}$ Also, if appropriate tools to assess intestinal dysbiosis ${ }^{19}$ are available, gut microbiome-targeted therapies could be also used only in those with altered microbial composition. Finally, drugs that improve dyslipidemia or decrease cardiovascular disease may be more effective for those patients with a more profound derangement of metabolic health such as patients with concurrent T2DM and NAFLD. In the latter population, one should also consider the potential benefits of antidiabetic drugs, such as pioglitazone and sodium-glucose cotransporter-2 (SGLT2) inhibitors, ${ }^{176,177}$ or the preferential use of the glucagon-like peptide 1 (GLP1) receptor agonists, such as liraglutide or semaglutide, with potentially beneficial impact on liver histology and cardiovascular outcomes in this particular patient population. ${ }^{178-181}$

Finally, consideration of psychosocial factors in the evaluation of any given patient is also relevant for NAFLD management and should be part of a comprehensive phenotyping approach. These factors might influence motivation to both adopt and sustain lifestyle changes. In this regard, necessary support to patients' efforts through health coaching and/or motivational interventions is key to successful adherence to any program aimed at naturalizing physical activity in daily life.

The impact of NAFLD heterogeneity in therapeutic clinical trials has been also emphasized recently. ${ }^{12,23}$ Precise stratification of patient cohorts, included in a given study, is a key when assessing therapeutic response in a complex disease such as NAFLD. The suboptimal responses observed in several clinical trials of different drugs may be in part due to the fact that disease heterogeneity was not taken into consideration. ${ }^{11,182}$ In their recent review on the matter Ampuero and Romero-Gomez concluded that clinical trial reporting for NAFLD has been suboptimal with no detailed mention of dysmetabolic comorbidities or proper recording of daily dietary and exercise habits. These authors suggest that future trials should carefully consider the presence of comorbidities expected to impact on the treatment response (trying to keep similar proportion of patients in the different arms of a given trial), as well as proper recording of alcohol intake and exercise both during the trial and not only at entry, which will improve capturing of the effects of these variables in treatment responses. Also, adoption of innovative trials designs to study novel NAFLD treatments, such as the adaptative, umbrella, or basket strategies, could be useful to improve trial efficiency. ${ }^{12,23}$ 


\section{Conclusion and Outlook}

NAFLD groups a heterogeneous patient population. The heterogeneity of NAFLD is reflected by the fact that some patients develop only steatosis, whereas others develop hepatocyte injury, steatohepatitis, and progressive liver fibrosis and, ultimately, cirrhosis. The interaction of different factors (e.g., sex, presence of one or more genetic variants, coexistence of different comorbidities such as obesity and T2DM, diverse microbiota composition, and various degrees of alcohol consumption) critically influence different disease pathways to determine disease subphenotypes with distinct natural history and prognosis and potentially different response to treatment. The recent proposal of NAFLD, renaming into MAFLD that sets positive criteria for diagnosis, ${ }^{24}$ has been generally welcomed by the scientific community, although debate is ongoing. ${ }^{25}$ The use of MAFLD may be a step forward in addressing disease heterogeneity by grouping a more homogenous patient population with more severe metabolic and liver disease (i.e., presence of significant fibrosis). ${ }^{183-185}$ In addition, differentiation of three subgroups (i.e., obese MAFLD, lean MAFLD, and diabetic MAFLD) that may have different prognosis and could be appraised differently, may be also relevant. However, further discussion is necessary and consensus needs to be reached on this matter. ${ }^{26,184}$

Taking into account, disease heterogeneity is relevant both clinically, to apply more individualized approaches in NAFLD diagnosis and treatment, and to drug development due to the need of including more homogeneous patient populations in clinical trials. In addition to clinical factors, availability of modern tools to define activation of DNL with metabolomics, development of accurate biomarkers of inflammatory, cell death or liver fibrosis pathways activation, better detection of presence of dysbiosis assisted by the use of microbiota signatures, or evaluation of genetic risk of cirrhosis or HCC with PRS would surely impact our ability to discriminate patients' subgroups and stratify them according to their risks of poor outcomes. This also would help to select the most appropriate treatment for a given patient following the precision medicine principles. ${ }^{13,15}$ Thus, in the future, it can be envisaged that precise phenotyping of pathogenic pathways would lead to select a specific drug ${ }^{10}$ or that a genetic treatment ${ }^{27,186}$ could be chosen based on global ethnic-specific genetic information. Also, it is likely that principles of precision nutrition ${ }^{187}$ and precision exercise ${ }^{188}$ would apply to patient management to increase the therapeutic efficacy of lifestyles changes in NAFLD management.

Indeed, further research is needed to improve our understanding of NAFLD heterogeneity. Building of large biobanks from well-characterized patient populations integrating clinical, genetic, biomarkers, and OMICs information is key to this end. The use of artificial intelligence approaches offers the opportunity to combine and decode this information to develop useful algorithms for patient stratification and management. A better definition of the dominant drivers of disease in patient subgroups would predictably impact on the development of more precision-targeted therapies for NAFLD.

\section{Main Concepts and Learning Points}

- Nonalcoholic fatty-liver disease (NAFLD) is an umbrella term that groups a heterogeneous patient population.

- NAFLD heterogeneity is influenced by multiple variables including age, sex, presence of one or more genetic variants, coexistence of different comorbidities, diverse microbiota composition, and various degrees of alcohol consumption among other factors.

- The heterogeneous nature of NAFLD results from a different hierarchy or trajectory of pathophysiological mechanisms that determine several disease subphenotypes with distinct natural history and prognosis and, eventually, different response to therapy.

- Proper consideration of NAFLD heterogeneity is relevant for both clinical practice to implement individualized medicine approaches, as well as for clinical research, to improve clinical trials efficiency.

- The recent renaming of NAFLD into MAFLD (i.e., metabolic [dysfunction]-associated fatty-liver disease) is a step forward in taking into account NAFLD heterogeneity by setting specific diagnostic criteria and grouping patients with disturbed metabolic health. However, more research is needed to refine discrimination of subtypes of the disease.

Abbreviations

ACC, acetyl-CoA carboxylase; APOB, apolipoprotein B; CaHSCs, central vein-associated HSCs; CRP, C-reactive protein; DAMPs, damage-associated molecular patterns; DNL, de novo lipogenesis; GCKR, glucokinase regulator; GWAS, genome-wide association studies; HCC, hepatocellular carcinoma; HSC, hepatic stellate cells; IL, interleukin; MBOAT7, membrane bound O-acyl transferase; MoMFs, monocyte-derived macrophages; NAFLD, nonalcoholic fatty-liver disease; NAFL, nonalcoholic fatty-liver; NASH, nonalcoholic steatohepatitis; NLRP3, NLR family pyrin domain containing 3; PaHSCs, portal vein-associated HSCs; PAMPs, pathogen-associated molecular patterns; PNPLA3, patatin-like phospholipase domaincontaining 3; PRS, polygenic risk scores; scRNA-seq, single cell RNA sequencing; TDM2, type 2 diabetes mellitus; TM6SF2: transmembrane 6 superfamily member 2; TNFa, tumor necrosis factor-a.

\section{Funding Sources}

This work was funded, in part, by grants from the Fondo Nacional De Ciencia y Tecnología de Chile (FONDECYT no.: 1191145 to M.A., no.:1200227 to J.P.A., and no.:1191183 to F.B.), the Comisión Nacional de Investigación, Ciencia y Tecnología (CONICYT, AFB170005, CARE, Chile, UC). M.A. is part of the European-Latin American ESCALON consortium funded by the European Union's Horizon 2020 Research and Innovation Program under grant agreement no. 825510 . A.E.F. was supported was by NIH grants R01 DK113592 and R01 AA024206. L.V. was supported by project grants from MyFirst Grant AIRC n.16888, Ricerca Finalizzata Ministero della Salute RF-2016-02364358 
("Impact of whole exome sequencing on the clinical management of patients with advanced nonalcoholic fatty-liver and cryptogenic-liver disease"), Ricerca corrente Fondazione IRCCS Ca' Granda Ospedale Maggiore Policlinico, the European Union (EU) Program Horizon 2020 (under grant agreement No. 777377) for the project LITMUS- "Liver Investigation: Testing Marker Utility in Steatohepatitis," Program "Photonics” under grant agreement "101016726" for the project "REVEAL: Neuronal microscopy for cell behavioural examination and manipulation," Gilead_IN-IT-989-5790 “Developing a model of care for risk stratification and management of diabetic patients with non-alcoholic fatty-liver disease (NAFLD)," Fondazione IRCCS Ca' Granda "Liver BIBLE" PR-0391, Fondazione IRCCS Ca' Granda core COVID-19 Biobank (RC100017A).

\section{Conflict of Interest}

The authors declare that they have no conflict of interest relevant to the present study. L.V. has received speaking fees from MSD, Gilead, AlfaSigma, and AbbVie, served as a consultant for Gilead, Pfizer, AstraZeneca, Novo Nordisk, Intercept, Diatech Pharmacogenetics and Ionis Pharmaceuticals, and received research grants from Gilead.

\section{References}

1 Younossi ZM, Koenig AB, Abdelatif D, Fazel Y, Henry L, Wymer M. Global epidemiology of nonalcoholic fatty liver disease-metaanalytic assessment of prevalence, incidence, and outcomes. Hepatology 2016;64(01):73-84

2 Cotter TG, Rinella M. Nonalcoholic fatty liver disease 2020: the state of the disease. Gastroenterology 2020;158(07): 1851-1864

3 Chalasani N, Younossi Z, Lavine JE, et al. The diagnosis and management of nonalcoholic fatty liver disease: Practice guidance from the American Association for the Study of Liver Diseases. Hepatology 2018;67(01):328-357

4 Huang DQ, El-Serag HB, Loomba R. Global epidemiology of NAFLD-related HCC: trends, predictions, risk factors and prevention. Nat Rev Gastroenterol Hepatol 2021;18(04): 223-238

5 Younossi ZM, Marchesini G, Pinto-Cortez H, Petta S. Epidemiology of nonalcoholic fatty liver disease and nonalcoholic steatohepatitis: implications for liver transplantation. Transplantation 2019;103(01):22-27

6 Estes C, Razavi H, Loomba R, Younossi Z, Sanyal AJ. Modeling the epidemic of nonalcoholic fatty liver disease demonstrates an exponential increase in burden of disease. Hepatology 2018;67 (01):123-133

7 Younossi ZM, Corey KE, Lim JK. AGA clinical practice update on lifestyle modification using diet and exercise to achieve weight loss in the management of nonalcoholic fatty liver disease: expert review. Gastroenterology 2021;160(03):912-918

8 Thorp A, Stine JG. Exercise as medicine: the impact of exercise training on nonalcoholic fatty liver disease. Curr Hepatol Rep 2020;19(04):402-411

9 Romero-Gómez M, Zelber-Sagi S, Trenell M. Treatment of NAFLD with diet, physical activity and exercise. J Hepatol 2017;67(04): 829-846

10 Vuppalanchi R, Noureddin M, Alkhouri N, Sanyal AJ. Therapeutic pipeline in nonalcoholic steatohepatitis. Nat Rev Gastroenterol Hepatol 2021
11 Ratziu V, Friedman SL. Why do so many NASH trials fail? Gastroenterology 2020:S0016-5085(20)30680-6

12 Ampuero J, Romero-Gomez M. Stratification of patients in NASH clinical trials: a pitfall for trial success. JHEP Rep 2020;2(05): 100148

13 Sookoian S, Pirola CJ. Precision medicine in nonalcoholic fatty liver disease: New therapeutic insights from genetics and systems biology. Clin Mol Hepatol 2020;26(04):461-475

14 Cespiati A, Youngson NA, Tourna A, Valenti L. Genetics and epigenetics in the clinic: precision medicine in the management of fatty liver disease. Curr Pharm Des 2020;26(10):998-1009

15 Lonardo A, Arab JP, Arrese M. Perspectives on precision medicine approaches to NAFLD diagnosis and management. Adv Ther 2021 (e-pub ahead of print). Doi: 10.1007/s12325-021-01690-1

16 Mauvais-Jarvis F, Bairey Merz N, Barnes PJ, et al. Sex and gender: modifiers of health, disease, and medicine. Lancet 2020;396 (10250):565-582

17 Trépo E, Valenti L. Update on NAFLD genetics: from new variants to the clinic. J Hepatol 2020;72(06):1196-1209

18 Byrne CD, Targher G. NAFLD: a multisystem disease. J Hepatol 2015;62(1, suppl):S47-S64

19 Sharpton SR, Schnabl B, Knight R, Loomba R. Current concepts, opportunities, and challenges of gut microbiome-based personalized medicine in nonalcoholic fatty liver disease. Cell Metab 2021;33(01):21-32

20 Arab JP, Arrese M, Shah VH. Gut microbiota in non-alcoholic fatty liver disease and alcohol-related liver disease: current concepts and perspectives. Hepatol Res 2020;50(04):407-418

21 Jiang L, Schnabl B. Gut microbiota in liver disease: what do we know and what do we not know? Physiology (Bethesda) 2020;35 (04):261-274

22 Idalsoaga F, Kulkarni AV, Mousa OY, Arrese M, Arab JP. Nonalcoholic fatty liver disease and alcohol-related liver disease: two intertwined entities. Front Med (Lausanne) 2020;7:448

23 Eslam M, Sanyal AJ, George JInternational Consensus Panel. MAFLD: a consensus-driven proposed nomenclature for metabolic associated fatty liver disease. Gastroenterology 2020;158 (07):1999-2014.e1

24 Eslam M, Newsome PN, Sarin SK, et al. A new definition for metabolic dysfunction-associated fatty liver disease: an international expert consensus statement. J Hepatol 2020;73(01): 202-209

25 Younossi ZM, Rinella ME, Sanyal AJ, et al. From NAFLD to MAFLD: implications of a premature change in terminology. Hepatology 2021;73(03):1194-1198

26 Ratziu V, Rinella M, Beuers U, et al. The times they are a-changin' (for NAFLD as well). J Hepatol 2020;73(06):1307-1309

27 Romeo S, Sanyal A, Valenti L. Leveraging human genetics to identify potential new treatments for fatty liver disease. Cell Metab 2020;31(01):35-45

28 Yki-Järvinen H. Non-alcoholic fatty liver disease as a cause and a consequence of metabolic syndrome. Lancet Diabetes Endocrinol 2014;2(11):901-910

29 Dongiovanni P, Petta S, Maglio C, et al. Transmembrane 6 superfamily member 2 gene variant disentangles nonalcoholic steatohepatitis from cardiovascular disease. Hepatology 2015; 61(02):506-514

30 Emdin CA, Haas M, Ajmera V, et al. Association of genetic variation with cirrhosis: a multi-trait genome-wide association and gene-environment interaction study. Gastroenterology 2021;160(05):1620-1633.e13

31 Jamialahmadi O, Mancina RM, Ciociola E, et al. Exome-wide association study on alanine aminotransferase identifies sequence variants in the GPAM and APOE associated with fatty liver disease. Gastroenterology 2021; 160(05):1634-1646.e7

32 Emdin CA, Haas ME, Khera AV, et al; Million Veteran Program. A missense variant in mitochondrial amidoxime reducing 
component 1 gene and protection against liver disease. PLoS Genet 2020;16(04):e1008629

33 Abul-Husn NS, Cheng X, Li AH, et al. A protein-truncating HSD17B13 variant and protection from chronic liver disease. N Engl J Med 2018;378(12):1096-1106

34 Dongiovanni P, Stender S, Pietrelli A, et al. Causal relationship of hepatic fat with liver damage and insulin resistance in nonalcoholic fatty liver. J Intern Med 2018;283(04):356-370

35 Bianco C, Jamialahmadi O, Pelusi S, et al. Non-invasive stratification of hepatocellular carcinoma risk in non-alcoholic fatty liver using polygenic risk scores. J Hepatol 2021;74(04):775-782

36 Gellert-Kristensen H, Richardson TG, Davey Smith G, Nordestgaard BG, Tybjaerg-Hansen A, Stender S. Combined effect of PNPLA3, TM6SF2, and HSD17B13 variants on risk of cirrhosis and hepatocellular carcinoma in the general population. Hepatology 2020;72(03):845-856

37 Stender S, Kozlitina J, Nordestgaard BG, Tybjærg-Hansen A, Hobbs HH, Cohen JC. Adiposity amplifies the genetic risk of fatty liver disease conferred by multiple loci. Nat Genet 2017;49(06): 842-847

38 Barata L, Feitosa MF, Bielak LF, et al. Insulin resistance exacerbates genetic predisposition to nonalcoholic fatty liver disease in individuals without diabetes. Hepatol Commun 2019;3(07): 894-907

39 Wei JL, Leung JC, Loong TC, et al. Prevalence and severity of nonalcoholic fatty liver disease in non-obese patients: a population study using proton-magnetic resonance spectroscopy. Am J Gastroenterol 2015;110(09):1306-1314, quiz 1315

40 Fracanzani AL, Petta S, Lombardi R, et al. Liver and cardiovascular damage in patients with lean nonalcoholic fatty liver disease, and association with visceral obesity. Clin Gastroenterol Hepatol 2017;15(10):1604-1611.e1

41 Valenti LVC, Baselli GA. Genetics of nonalcoholic fatty liver disease: a 2018 update. Curr Pharm Des 2018;24(38):4566-4573

42 Valenti L, Nobili V. PNPLA3 I148M polymorphism and liver damage in obese children. J Pediatr 2011;159(05):876

43 Valenti L, Rumi M, Galmozzi E, et al. Patatin-like phospholipase domain-containing 3 I148M polymorphism, steatosis, and liver damage in chronic hepatitis C. Hepatology 2011;53(03):791-799

44 Dongiovanni P, Donati B, Fares R, et al. PNPLA3 I148M polymorphism and progressive liver disease. World J Gastroenterol 2013; 19(41):6969-6978

45 Pelusi S, Baselli G, Pietrelli A, et al. Rare pathogenic variants predispose to hepatocellular carcinoma in nonalcoholic fatty liver disease. Sci Rep 2019;9(01):3682

46 Cefalù AB, Pirruccello JP, Noto D, et al. A novel APOB mutation identified by exome sequencing cosegregates with steatosis, liver cancer, and hypocholesterolemia. Arterioscler Thromb Vasc Biol 2013;33(08):2021-2025

47 Valenti L, Romeo S. Editorial: new insights into the relationship between the intestine and non-alcoholic fatty liver-is "fatty gut" involved in disease progression? Aliment Pharmacol Ther 2017; 46(03):377-378

48 Liu Z, Zhang Y, Graham S, et al. Causal relationships between NAFLD, T2D and obesity have implications for disease subphenotyping. J Hepatol 2020;73(02):263-276

49 Speliotes EK, Yerges-Armstrong LM, Wu J, et al; NASH CRN GIANT Consortium MAGIC Investigators GOLD Consortium. Genomewide association analysis identifies variants associated with nonalcoholic fatty liver disease that have distinct effects on metabolic traits. PLoS Genet 2011;7(03):e1001324

50 Dongiovanni P, Petta S, Mannisto V, et al. Statin use and nonalcoholic steatohepatitis in at risk individuals. J Hepatol 2015;63 (03):705-712

51 Arab JP, Arrese M, Trauner M. Recent insights into the pathogenesis of nonalcoholic fatty liver disease. Annu Rev Pathol 2018; 13:321-350
52 Brunt EM, Wong VW, Nobili V, et al. Nonalcoholic fatty liver disease. Nat Rev Dis Primers 2015;1:15080

53 Friedman SL, Neuschwander-Tetri BA, Rinella M, Sanyal AJ. Mechanisms of NAFLD development and therapeutic strategies. Nat Med 2018;24(07):908-922

54 Sookoian S, Pirola CJ, Valenti L, Davidson NO. Genetic pathways in nonalcoholic fatty liver disease: insights from systems biology. Hepatology 2020;72(01):330-346

55 Bence KK, Birnbaum MJ. Metabolic drivers of non-alcoholic fatty liver disease. Mol Metab 2020 (e-pub ahead of print). Doi: 10.1016/j.molmet.2020.101143

56 Buzzetti E, Pinzani M, Tsochatzis EA. The multiple-hit pathogenesis of non-alcoholic fatty liver disease (NAFLD). Metabolism 2016;65(08):1038-1048

57 Tilg H, Adolph TE, Moschen AR. Multiple parallel hits hypothesis in nonalcoholic fatty liver disease - revisited after a decade. Hepatology 2021;73(02):833-842

58 Lambert JE, Ramos-Roman MA, Browning JD, Parks EJ. Increased de novo lipogenesis is a distinct characteristic of individuals with nonalcoholic fatty liver disease. Gastroenterology 2014; 146(03):726-735

59 Smith GI, Shankaran M, Yoshino M, et al. Insulin resistance drives hepatic de novo lipogenesis in nonalcoholic fatty liver disease. J Clin Invest 2020;130(03):1453-1460

60 Schuster S, Cabrera D, Arrese M, Feldstein AE. Triggering and resolution of inflammation in NASH. Nat Rev Gastroenterol Hepatol 2018;15(06):349-364

61 Parthasarathy G, Revelo X, Malhi H. Pathogenesis of nonalcoholic steatohepatitis: an overview. Hepatol Commun 2020;4(04): 478-492

62 Buyco DG, Martin J, Jeon S, Hooks R, Lin C, Carr R. Experimental models of metabolic and alcoholic fatty liver disease. World J Gastroenterol 2021;27(01):1-18

63 Ipsen DH, Lykkesfeldt J, Tveden-Nyborg P. Animal models of fibrosis in nonalcoholic steatohepatitis: do they reflect human disease? Adv Nutr 2020;11(06):1696-1711

64 Pramfalk C, Pavlides M, Banerjee R, et al. Sex-specific differences in hepatic fat oxidation and synthesis may explain the higher propensity for NAFLD in men. J Clin Endocrinol Metab 2015;100 (12):4425-4433

65 Paglialunga S, Dehn CA. Clinical assessment of hepatic de novo lipogenesis in non-alcoholic fatty liver disease. Lipids Health Dis 2016;15(01):159

66 Fernández-Mincone T, Contreras-Briceño F, Espinosa-Ramírez $\mathrm{M}$, et al. Nonalcoholic fatty liver disease and sarcopenia: pathophysiological connections and therapeutic implications. Expert Rev Gastroenterol Hepatol 2020;14(12):1141-1157

67 Cabrera D, Ruiz A, Cabello-Verrugio C, et al. Diet-induced nonalcoholic fatty liver disease is associated with sarcopenia and decreased serum insulin-like growth factor-1. Dig Dis Sci 2016;61(11):3190-3198

68 Chakravarthy MV, Siddiqui MS, Forsgren MF, Sanyal AJ. Harnessing muscle-liver crosstalk to treat nonalcoholic steatohepatitis. Front Endocrinol (Lausanne) 2020;11:592373

69 Kashiwagi K, Takayama M, Fukuhara K, et al. A significant association of non-obese non-alcoholic fatty liver disease with sarcopenic obesity. Clin Nutr ESPEN 2020;38:86-93

70 Cai J, Zhang XJ, Li H. The role of innate immune cells in nonalcoholic steatohepatitis. Hepatology 2019;70(03):1026-1037

71 Arrese M, Cabrera D, Kalergis AM, Feldstein AE. Innate immunity and inflammation in NAFLD/NASH. Dig Dis Sci 2016;61(05): 1294-1303

72 Chu H, Duan Y, Yang L, Schnabl B. Small metabolites, possible big changes: a microbiota-centered view of non-alcoholic fatty liver disease. Gut 2019;68(02):359-370

73 Porras D, Nistal E, Martínez-Flórez S, González-Gallego J, GarcíaMediavilla MV, Sánchez-Campos S. Intestinal microbiota 
modulation in obesity-related non-alcoholic fatty liver disease. Front Physiol 2018;9:1813

74 Tripathi A, Debelius J, Brenner DA, et al. The gut-liver axis and the intersection with the microbiome. Nat Rev Gastroenterol Hepatol 2018;15(07):397-411

75 Candia R, Ruiz A, Torres-Robles R, Chávez-Tapia N, MéndezSánchez N, Arrese M. Risk of non-alcoholic fatty liver disease in patients with psoriasis: a systematic review and meta-analysis. J Eur Acad Dermatol Venereol 2015;29(04):656-662

76 Heitmann J, Frings VG, Geier A, Goebeler M, Kerstan A. Nonalcoholic fatty liver disease and psoriasis - is there a shared proinflammatory network? J Dtsch Dermatol Ges 2021

77 Damiani G, Leone S, Fajgenbaum K, et al. Nonalcoholic fatty liver disease prevalence in an Italian cohort of patients with hidradenitis suppurativa: a multi-center retrospective analysis. World J Hepatol 2019;11(04):391-401

78 van der Voort EA, Koehler EM, Dowlatshahi EA, et al. Psoriasis is independently associated with nonalcoholic fatty liver disease in patients 55 years old or older: results from a population-based study. J Am Acad Dermatol 2014;70(03):517-524

79 van der Voort EA, Koehler EM, Nijsten T, et al. Increased prevalence of advanced liver fibrosis in patients with psoriasis: a cross-sectional analysis from the rotterdam study. Acta Derm Venereol 2016;96(02):213-217

80 Durán-Vian C, Arias-Loste MT, Hernández JL, et al. High prevalence of non-alcoholic fatty liver disease among hidradenitis suppurativa patients independent of classic metabolic risk factors. J Eur Acad Dermatol Venereol 2019;33(11):2131-2136

81 Prussick RB, Miele L. Nonalcoholic fatty liver disease in patients with psoriasis: a consequence of systemic inflammatory burden? Br J Dermatol 2018;179(01):16-29

82 He B, Wu L, Xie W, et al. The imbalance of Th17/Treg cells is involved in the progression of nonalcoholic fatty liver disease in mice. BMC Immunol 2017;18(01):33

83 Luedde T, Kaplowitz N, Schwabe RF. Cell death and cell death responses in liver disease: mechanisms and clinical relevance. Gastroenterology 2014;147(04):765-783.e4

84 Wree A, Holtmann TM, Inzaugarat ME, Feldstein AE. Novel drivers of the inflammatory response in liver injury and fibrosis. Semin Liver Dis 2019;39(03):275-282

85 Wree A, Eguchi A, McGeough MD, et al. NLRP3 inflammasome activation results in hepatocyte pyroptosis, liver inflammation, and fibrosis in mice. Hepatology 2014;59(03):898-910

86 Wree A, McGeough MD, Peña CA, et al. NLRP3 inflammasome activation is required for fibrosis development in NAFLD. J Mol Med (Berl) 2014;92(10):1069-1082

87 Krenkel O, Tacke F. Liver macrophages in tissue homeostasis and disease. Nat Rev Immunol 2017;17(05):306-321

88 Krenkel O, Hundertmark J, Abdallah AT, et al. Myeloid cells in liver and bone marrow acquire a functionally distinct inflammatory phenotype during obesity-related steatohepatitis. Gut 2020;69(03):551-563

89 Chu AL, Schilling JD, King KR, Feldstein AE. The power of singlecell analysis for the study of liver pathobiology. Hepatology 2021;73(01):437-448

90 Xiong X, Kuang H, Ansari S, et al. Landscape of intercellular crosstalk in healthy and NASH liver revealed by single-cell secretome gene analysis. Mol Cell 2019;75(03):644-660.e5

91 Daemen S, Gainullina A, Kalugotla G, et al. Dynamic shifts in the composition of resident and recruited macrophages influence tissue remodeling in NASH. Cell Rep 2021;34(02):108626

92 Tran S, Baba I, Poupel L, et al. Impaired Kupffer cell self-renewal alters the liver response to lipid overload during non-alcoholic steatohepatitis. Immunity 2020;53(03):627-640.e5

93 Vilar-Gomez E, Calzadilla-Bertot L, Wai-Sun Wong V, et al. Fibrosis severity as a determinant of cause-specific mortality in patients with advanced nonalcoholic fatty liver disease: a multi-national cohort study. Gastroenterology 2018;155(02):443-457.e17
94 Ekstedt M, Hagström H, Nasr P, et al. Fibrosis stage is the strongest predictor for disease-specific mortality in NAFLD after up to 33 years of follow-up. Hepatology 2015;61(05):1547-1554

95 Kisseleva T, Brenner D. Molecular and cellular mechanisms of liver fibrosis and its regression. Nat Rev Gastroenterol Hepatol 2021;18(03):151-166

96 Schwabe RF, Tabas I, Pajvani UB. Mechanisms of fibrosis development in nonalcoholic steatohepatitis. Gastroenterology 2020; 158(07):1913-1928

97 Krenkel O, Hundertmark J, Ritz TP, Weiskirchen R, Tacke F. Single cell RNA sequencing identifies subsets of hepatic stellate cells and myofibroblasts in liver fibrosis. Cells 2019;8(05):E503

98 Dobie R, Wilson-Kanamori JR, Henderson BEP, et al. Single-cell transcriptomics uncovers zonation of function in the mesenchyme during liver fibrosis. Cell Rep 2019;29(07):1832-1847.e8

99 Lonardo A, Nascimbeni F, Ballestri S, et al. Sex differences in nonalcoholic fatty liver disease: state of the art and identification of research gaps. Hepatology 2019;70(04):1457-1469

100 Bertolotti M, Lonardo A, Mussi C, et al. Nonalcoholic fatty liver disease and aging: epidemiology to management. World J Gastroenterol 2014;20(39):14185-14204

101 Vittorio J, Lavine JE. Recent advances in understanding and managing pediatric nonalcoholic fatty liver disease. F1000 Res 2020;9:9

102 Golabi P, Paik J, Reddy R, Bugianesi E, Trimble G, Younossi ZM. Prevalence and long-term outcomes of non-alcoholic fatty liver disease among elderly individuals from the United States. BMC Gastroenterol 2019;19(01):56

103 Yang JD, Abdelmalek MF, Pang H, et al. Gender and menopause impact severity of fibrosis among patients with nonalcoholic steatohepatitis. Hepatology 2014;59(04):1406-1414

104 Petroni ML, Brodosi L, Bugianesi E, Marchesini G. Management of non-alcoholic fatty liver disease. BMJ 2021;372:m4747

105 Kanwal F, Kramer JR, Li L, et al. Effect of metabolic traits on the risk of cirrhosis and hepatocellular cancer in nonalcoholic fatty liver disease. Hepatology 2020;71(03):808-819

106 Younossi ZM, Golabi P, de Avila L, et al. The global epidemiology of NAFLD and NASH in patients with type 2 diabetes: A systematic review and meta-analysis. J Hepatol 2019;71(04): 793-801

107 Jarvis H, Craig D, Barker R, et al. Metabolic risk factors and incident advanced liver disease in non-alcoholic fatty liver disease (NAFLD): A systematic review and meta-analysis of population-based observational studies. PLoS Med 2020;17 (04):e1003100

108 Arab JP, Dirchwolf M, Álvares-da-Silva MR, et al. Latin American Association for the study of the liver (ALEH) practice guidance for the diagnosis and treatment of non-alcoholic fatty liver disease. Ann Hepatol 2020;19(06):674-690

109 Tsochatzis EA, Newsome PN. Non-alcoholic fatty liver disease and the interface between primary and secondary care. Lancet Gastroenterol Hepatol 2018;3(07):509-517

110 Lu FB, Hu ED, Xu LM, et al. The relationship between obesity and the severity of non-alcoholic fatty liver disease: systematic review and meta-analysis. Expert Rev Gastroenterol Hepatol 2018;12(05):491-502

111 Younes R, Govaere O, Petta S, et al. Caucasian lean subjects with non-alcoholic fatty liver disease share long-term prognosis of non-lean: time for reappraisal of BMI-driven approach? Gut 2021 (e-pub ahead of print). Doi: 10.1136/gutjnl-2020-322564

112 Ye Q Zou B, Yeo YH, et al. Global prevalence, incidence, and outcomes of non-obese or lean non-alcoholic fatty liver disease: a systematic review and meta-analysis. Lancet Gastroenterol Hepatol 2020;5(08):739-752

113 Lu FB, Zheng KI, Rios RS, Targher G, Byrne CD, Zheng MH. Global epidemiology of lean non-alcoholic fatty liver disease: a systematic review and meta-analysis. J Gastroenterol Hepatol 2020;35 (12):2041-2050 
114 Younes R, Bugianesi E. NASH in lean individuals. Semin Liver Dis 2019;39(01):86-95

115 Golabi P, Paik J, Fukui N, Locklear CT, de Avilla L, Younossi ZM. Patients with lean nonalcoholic fatty liver disease are metabolically abnormal and have a higher risk for mortality. Clin Diabetes 2019;37(01):65-72

116 Younossi Z, Anstee QM, Marietti M, et al. Global burden of NAFLD and NASH: trends, predictions, risk factors and prevention. Nat Rev Gastroenterol Hepatol 2018;15(01):11-20

117 Le MH, Yeo YH, Cheung R, Wong VW, Nguyen MH. Ethnic influence on nonalcoholic fatty liver disease prevalence and lack of disease awareness in the United States, 2011-2016. J Intern Med 2020;287(06):711-722

118 Lonardo A, Suzuki A. Focus on Clinical Aspects and Implications for Practice and Translational Research. Sexual dimorphism of NAFLD in adults. J Clin Med 2020;9(05):E1278

119 Balakrishnan M, Patel P, Dunn-Valadez S, et al. Women have a lower risk of nonalcoholic fatty liver disease but a higher risk of progression vs men: a systematic review and meta-analysis. Clin Gastroenterol Hepatol 2021;19(01):61-71.e15

120 Parikh MP, Gupta NM, McCullough AJ. Obstructive sleep apnea and the liver. Clin Liver Dis 2019;23(02):363-382

121 Hernández A, Geng Y, Sepúlveda R, et al. Chemical hypoxia induces pro-inflammatory signals in fat-laden hepatocytes and contributes to cellular crosstalk with Kupffer cells through extracellular vesicles. Biochim Biophys Acta Mol Basis Dis 2020;1866(06):165753

122 Mesarwi OA, Loomba R, Malhotra A. Obstructive sleep apnea, hypoxia, and nonalcoholic fatty liver disease. Am J Respir Crit Care Med 2019;199(07):830-841

123 Rosato V, Masarone M, Dallio M, Federico A, Aglitti A, Persico M. NAFLD and extra-hepatic comorbidities: current evidence on a multi-organ metabolic syndrome. Int J Environ Res Public Health 2019;16(18):E3415

124 Matteoni CA, Younossi ZM, Gramlich T, Boparai N, Liu YC, McCullough AJ. Nonalcoholic fatty liver disease: a spectrum of clinical and pathological severity. Gastroenterology 1999;116 (06):1413-1419

125 Pelusi S, Cespiati A, Rametta R, et al. Prevalence and risk factors of significant fibrosis in patients with nonalcoholic fatty liver without steatohepatitis. Clin Gastroenterol Hepatol 2019;17 (11):2310-2319.e6

126 Kleiner DE, Brunt EM, Wilson LA, et al; Nonalcoholic Steatohepatitis Clinical Research Network. Association of histologic disease activity with progression of nonalcoholic fatty liver disease. JAMA Netw Open 2019;2(10):e1912565

127 Ekstedt M, Franzén LE, Mathiesen UL, Kechagias S. Low clinical relevance of the nonalcoholic fatty liver disease activity score (NAS) in predicting fibrosis progression. Scand J Gastroenterol 2012;47(01):108-115

128 Pais R, Charlotte F, Fedchuk L, et al; LIDO Study Group. A systematic review of follow-up biopsies reveals disease progression in patients with non-alcoholic fatty liver. J Hepatol 2013;59 (03):550-556

129 Wong VW, Wong GL, Choi PC, et al. Disease progression of nonalcoholic fatty liver disease: a prospective study with paired liver biopsies at 3 years. Gut 2010;59(07):969-974

130 Argo CK, Northup PG, Al-Osaimi AM, Caldwell SH. Systematic review of risk factors for fibrosis progression in non-alcoholic steatohepatitis. J Hepatol 2009;51(02):371-379

131 Singh S, Allen AM, Wang Z, Prokop LJ, Murad MH, Loomba R. Fibrosis progression in nonalcoholic fatty liver vs nonalcoholic steatohepatitis: a systematic review and meta-analysis of paired-biopsy studies. Clin Gastroenterol Hepatol 2015;13 (04):643-654.e1, 9, quiz e39-e40

132 Cariou B, Byrne CD, Loomba R, Sanyal AJ. Nonalcoholic fatty liver disease as a metabolic disease in humans: a literature review. Diabetes Obes Metab 2021;23(05):1069-1083
133 McPherson S, Hardy T, Henderson E, Burt AD, Day CP, Anstee QM. Evidence of NAFLD progression from steatosis to fibrosingsteatohepatitis using paired biopsies: implications for prognosis and clinical management. J Hepatol 2015;62(05): $1148-1155$

134 Teli MR, James OF, Burt AD, Bennett MK, Day CP. The natural history of nonalcoholic fatty liver: a follow-up study. Hepatology 1995;22(06):1714-1719

135 Powell EE, Cooksley WG, Hanson R, Searle J, Halliday JW, Powell LW. The natural history of nonalcoholic steatohepatitis: a follow-up study of forty-two patients for up to 21 years. Hepatology 1990;11(01):74-80

136 Ludwig J, Viggiano TR, McGill DB, Oh BJ. Nonalcoholic steatohepatitis: Mayo Clinic experiences with a hitherto unnamed disease. Mayo Clin Proc 1980;55(07):434-438

137 Adler M, Schaffner F. Fatty liver hepatitis and cirrhosis in obese patients. Am J Med 1979;67(05):811-816

138 Wanless IR, Lentz JS. Fatty liver hepatitis (steatohepatitis) and obesity: an autopsy study with analysis of risk factors. Hepatology 1990;12(05):1106-1110

139 Angulo P, Kleiner DE, Dam-Larsen S, et al. Liver fibrosis, but no other histologic features, is associated with long-term outcomes of patients with nonalcoholic fatty liver disease. Gastroenterology 2015;149(02):389-97.e10

140 Younossi ZM, Stepanova M, Rafiq N, et al. Pathologic criteria for nonalcoholic steatohepatitis: interprotocol agreement and ability to predict liver-related mortality. Hepatology 2011;53(06): 1874-1882

141 Kim D, Kim WR, Kim HJ, Therneau TM. Association between noninvasive fibrosis markers and mortality among adults with nonalcoholic fatty liver disease in the United States. Hepatology 2013;57(04):1357-1365

142 Vuppalanchi R, Jain AK, Deppe R, et al. Relationship between changes in serum levels of keratin 18 and changes in liver histology in children and adults with nonalcoholic fatty liver disease. Clin Gastroenterol Hepatol 2014;12(12):2121-30.e1, 2

143 Feldstein AE, Wieckowska A, Lopez AR, Liu YC, Zein NN, McCullough AJ. Cytokeratin-18 fragment levels as noninvasive biomarkers for nonalcoholic steatohepatitis: a multicenter validation study. Hepatology 2009;50(04):1072-1078

144 Wong VW, Hui AY, Tsang SW, et al. Metabolic and adipokine profile of Chinese patients with nonalcoholic fatty liver disease. Clin Gastroenterol Hepatol 2006;4(09):1154-1161

145 Musso G, Gambino R, Durazzo M, et al. Adipokines in NASH: postprandial lipid metabolism as a link between adiponectin and liver disease. Hepatology 2005;42(05):1175-1183

146 Hui JM, Hodge A, Farrell GC, Kench JG, Kriketos A, George J. Beyond insulin resistance in NASH: TNF-alpha or adiponectin? Hepatology 2004;40(01):46-54

147 Angulo P, Hui JM, Marchesini G, et al. The NAFLD fibrosis score: a noninvasive system that identifies liver fibrosis in patients with NAFLD. Hepatology 2007;45(04):846-854

148 Sterling RK, Lissen E, Clumeck N, et al; APRICOT Clinical Investigators. Development of a simple noninvasive index to predict significant fibrosis in patients with HIV/HCV coinfection. Hepatology 2006;43(06):1317-1325

149 Angulo P, Bugianesi E, Bjornsson ES, et al. Simple noninvasive systems predict long-term outcomes of patients with nonalcoholic fatty liver disease. Gastroenterology 2013;145(04):782-9. e4

150 McPherson S, Stewart SF, Henderson E, Burt AD, Day CP. Simple non-invasive fibrosis scoring systems can reliably exclude advanced fibrosis in patients with non-alcoholic fatty liver disease. Gut 2010;59(09):1265-1269

151 Harrison SA, Oliver D, Arnold HL, Gogia S, Neuschwander-Tetri BA. Development and validation of a simple NAFLD clinical scoring system for identifying patients without advanced disease. Gut 2008;57(10):1441-1447 
152 Ampuero J, Pais R, Aller R, et al; HEPAmet Registry. Development and validation of hepamet fibrosis scoring system-a simple, noninvasive test to identify patients with nonalcoholic fatty liver disease with advanced fibrosis. Clin Gastroenterol Hepatol 2020;18(01):216-225.e5

153 Castera L, Friedrich-Rust M, Loomba R. Noninvasive assessment of liver disease in patients with nonalcoholic fatty liver disease. Gastroenterology 2019;156(05):1264-1281.e4

154 Chen J, Talwalkar JA, Yin M, Glaser KJ, Sanderson SO, Ehman RL. Early detection of nonalcoholic steatohepatitis in patients with nonalcoholic fatty liver disease by using MR elastography. Radiology 2011;259(03):749-756

155 Noureddin M, Lam J, Peterson MR, et al. Utility of magnetic resonance imaging versus histology for quantifying changes in liver fat in nonalcoholic fatty liver disease trials. Hepatology 2013;58(06):1930-1940

156 European Association for Study of Liver Asociacion Latinoamericana para el Estudio del Higado. EASL-ALEH clinical practice guidelines: non-invasive tests for evaluation of liver disease severity and prognosis. J Hepatol 2015;63(01):237-264

157 Tapper EB, Challies T, Nasser I, Afdhal NH, Lai M. The performance of vibration controlled transient elastography in a US cohort of patients with nonalcoholic fatty liver disease. Am J Gastroenterol 2016;111(05):677-684

158 Chen J, Yin M, Talwalkar JA, et al. Diagnostic performance of MR elastography and vibration-controlled transient elastography in the detection of hepatic fibrosis in patients with severe to morbid obesity. Radiology 2017;283(02):418-428

159 Adams LA, Chan WK. Noninvasive tests in the assessment of NASH and NAFLD fibrosis: now and into the future. Semin Liver Dis 2020;40(04):331-338

160 Loomba R, Adams LA. Advances in non-invasive assessment of hepatic fibrosis. Gut 2020;69(07):1343-1352

161 Subramanian M, Wojtusciszyn A, Favre L, et al. Precision medicine in the era of artificial intelligence: implications in chronic disease management. J Transl Med 2020;18(01):472

162 Sanyal AJ. Past, present and future perspectives in nonalcoholic fatty liver disease. Nat Rev Gastroenterol Hepatol 2019;16(06): 377-386

163 Arrese M, Barrera F, Triantafilo N, Arab JP. Concurrent nonalcoholic fatty liver disease and type 2 diabetes: diagnostic and therapeutic considerations. Expert Rev Gastroenterol Hepatol 2019;13(09):849-866

164 Paik JM, Deshpande R, Golabi P, Younossi I, Henry L, Younossi ZM. The impact of modifiable risk factors on the long-term outcomes of non-alcoholic fatty liver disease. Aliment Pharmacol Ther 2020;51(02):291-304

165 Saiman Y, Hooks R, Carr RM. High-risk groups for non-alcoholic fatty liver and non-alcoholic steatohepatitis development and progression. Curr Hepatol Rep 2020;19:412-419

166 Koo BK, Joo SK, Kim D, et al. Development and validation of a scoring system, based on genetic and clinical factors, to determine risk of steatohepatitis in asian patients with nonalcoholic fatty liver disease. Clin Gastroenterol Hepatol 2020;18(11): 2592-2599.e10

167 European Association for the Study of the Liver (EASL) European Association for the Study of Diabetes (EASD) European Association for the Study of Obesity (EASO) EASL-EASD-EASO Clinical Practice Guidelines for the management of non-alcoholic fatty liver disease. J Hepatol 2016;64(06):1388-1402

168 Vilar-Gomez E, Martinez-Perez Y, Calzadilla-Bertot L, et al. Weight loss through lifestyle modification significantly reduces features of nonalcoholic steatohepatitis. Gastroenterology 2015; 149(02):367-78.e5, quiz e14-e15

169 Bhogal MS, Langford R. Gender differences in weight loss; evidence from a NHS weight management service. Public Health 2014;128(09):811-813
170 Shen J, Wong GL, Chan HL, et al. PNPLA3 gene polymorphism and response to lifestyle modification in patients with nonalcoholic fatty liver disease. J Gastroenterol Hepatol 2015;30(01):139-146

171 Krawczyk M, Jiménez-Agüero R, Alustiza JM, et al. PNPLA3 p. I148M variant is associated with greater reduction of liver fat content after bariatric surgery. Surg Obes Relat Dis 2016;12(10): 1838-1846

172 Luukkonen PK, Qadri S, Lehtimäki TE, et al. The PNPLA3-I148M variant confers an antiatherogenic lipid profile in insulinresistant patients. J Clin Endocrinol Metab 2021;106(01): e300-e315

173 Bianco C, Jamialahmadi O, Pelusi S, et al. Non-invasive stratification of hepatocellular carcinoma risk in non-alcoholic fatty liver using polygenic risk scores. J Hepatol 2021;74(04): 775-782

174 Mato JM, Alonso C, Noureddin M, Lu SC. Biomarkers and subtypes of deranged lipid metabolism in non-alcoholic fatty liver disease. World J Gastroenterol 2019;25(24):3009-3020

175 Alonso C, Fernández-Ramos D, Varela-Rey M, et al. Metabolomic identification of subtypes of nonalcoholic steatohepatitis. Gastroenterology 2017;152(06):1449-1461.e7

176 Xing B, Zhao Y, Dong B, Zhou Y, Lv W, Zhao W. Effects of sodiumglucose cotransporter 2 inhibitors on non-alcoholic fatty liver disease in patients with type 2 diabetes: a meta-analysis of randomized controlled trials. J Diabetes Investig 2020;11(05): $1238-1247$

177 Blazina I, Selph S. Diabetes drugs for nonalcoholic fatty liver disease: a systematic review. Syst Rev 2019;8(01):295

178 Lv X, Dong Y, Hu L, Lu F, Zhou C, Qin S. Glucagon-like peptide-1 receptor agonists (GLP-1 RAs) for the management of nonalcoholic fatty liver disease (NAFLD): a systematic review. Endocrinol Diabetes Metab 2020;3(03):e00163

179 Mantovani A, Byrne CD, Scorletti E, Mantzoros CS, Targher G. Efficacy and safety of anti-hyperglycaemic drugs in patients with non-alcoholic fatty liver disease with or without diabetes: An updated systematic review of randomized controlled trials. Diabetes Metab 2020;46(06):427-441

180 Mantovani A, Petracca G, Csermely A, Beatrice G, Targher G. Sodium-Glucose cotransporter-2 inhibitors for treatment of nonalcoholic fatty liver disease: a meta-analysis of randomized controlled trials. Metabolites 2020;11(01):22

181 Wong C, Yaow CYL, Ng CH, et al. Sodium-glucose co-transporter 2 inhibitors for non-alcoholic fatty liver disease in asian patients with type 2 diabetes: a meta-analysis. Front Endocrinol (Lausanne) 2021;11:609135

182 Drenth JPH, Schattenberg JM. The nonalcoholic steatohepatitis (NASH) drug development graveyard: established hurdles and planning for future success. Expert Opin Investig Drugs 2020;29 (12):1365-1375

183 Lin S, Huang J, Wang M, et al. Comparison of MAFLD and NAFLD diagnostic criteria in real world. Liver Int 2020;40(09): 2082-2089

184 Wai-Sun Wong V, Kanwal F. On the proposed definition of metabolic-associated fatty liver disease. Clin Gastroenterol Hepatol 2021;19(05):865-870

185 Yamamura S, Eslam M, Kawaguchi T, et al. MAFLD identifies patients with significant hepatic fibrosis better than NAFLD. Liver Int 2020;40(12):3018-3030

186 Lindén D, Ahnmark A, Pingitore P, et al. Pnpla3 silencing with antisense oligonucleotides ameliorates nonalcoholic steatohepatitis and fibrosis in Pnpla3 I148M knock-in mice. Mol Metab 2019;22:49-61

187 Kaiser J. NIH's 'precision nutrition' bet aims for individualized diets. Science 2021;371(6529):552

188 Ross R, Goodpaster BH, Koch LG, et al. Precision exercise medicine: understanding exercise response variability. $\mathrm{Br} \mathrm{J}$ Sports Med 2019;53(18):1141-1153 